\title{
Individual Channel Estimation in a Diamond Relay Network Using Relay-Assisted Training
}

\author{
Xianwen He, Gaoqi Dou, and Jun Gao \\ College of Electronic Engineering, Naval University of Engineering, Wuhan 430033, China \\ Correspondence should be addressed to Gaoqi Dou; hjgcqq@163.com
}

Received 12 April 2017; Revised 18 September 2017; Accepted 1 October 2017; Published 8 November 2017

Academic Editor: Wanggen Wan

Copyright (C) 2017 Xianwen He et al. This is an open access article distributed under the Creative Commons Attribution License, which permits unrestricted use, distribution, and reproduction in any medium, provided the original work is properly cited.

\begin{abstract}
We consider the training design and channel estimation in the amplify-and-forward (AF) diamond relay network. Our strategy is to transmit the source training in time-multiplexing (TM) mode while each relay node superimposes its own relay training over the amplified received data signal without bandwidth expansion. The principal challenge is to obtain accurate channel state information (CSI) of second-hop link due to the multiaccess interference (MAI) and cooperative data interference (CDI). To maintain the orthogonality between data and training, a modified relay-assisted training scheme is proposed to migrate the CDI, where some of the cooperative data at the relay are discarded to accommodate relay training. Meanwhile, a couple of optimal zero-correlation zone (ZCZ) relay-assisted sequences are designed to avoid MAI. At the destination node, the received signals from the two relay nodes are combined to achieve spatial diversity and enhanced data reliability. The simulation results are presented to validate the performance of the proposed schemes.
\end{abstract}

\section{Introduction}

To combat the effects of multipath fading in wireless networks, relay cooperative communication is proposed to generate a virtual multiple-antenna network by sharing antennas $[1,2]$. This topic has been the subject of intensive research due to its potential for providing spatial diversity and coverage extension without the limitation of hardware complexity [3]. The diamond relay network is one of the special cases of the multiple-relay networks. Specifically, with only two relays utilized, the diamond relay network can yield diversity benefits to combat fading and be simple enough to design the transmission protocol and efficient scheduling [4]. Moreover, as compared to three-node network with a single relay utilized, the most advantage of the diamond relay network is that different relays can transmit and receive at the same time, which in turn translates to gains due to spatial reuse [5]. Many applications practical in diamond relay network have been studied, for example, the optimal power allocation $[6,7]$, the optimal opportunistic relay scheme [8-11], and the optimal position selection of relays $[4,5]$. In essence, a diamond relay network is a cascade of two single-hop links consisting of the broadcast channel and the multiple-access channel. However, purely knowing the cascaded channel is insufficient to support the above optimal design in diamond relay network. As a result, channel estimation problems are generally more challenging in diamond relay networks than in three-node relay networks.

With regard to an amplify-and-forward- (AF-) based relay cooperation system, most research has focused on acquiring the cascaded channel state information (CSI). The authors in [12] designed a cyclic-orthogonal training sequence and presented a practical estimation algorithm for a cascaded channel. In [13], the authors derived necessary and sufficient conditions for a relay amplifying matrix in MultiInput Multi-Output (MIMO) systems. However, the schemes presented in $[12,13]$ cannot be adopted for estimating an individual channel. For most applications, the CSI of an individual link is indispensable at the receiver to perform signal retrieving and system optimization $[14,15]$. Recently, it has been shown in [16] that a multiuser receiver can be used to blindly estimate the channel matrices associated with both individual links. Unfortunately, it is difficult to obtain an instantaneous CSI. The subsequent work in [17] 
had expanded the superimposed training scheme presented in [18] to the area of multirelay networks. The authors derived optimal pilot symbol designs including both modification diagonal matrix and relay superimposed pilot symbol. Nevertheless, the orthogonal constraint among the source pilot, superimposed pilot, and the modification matrix demands an additional spending and a protocol to coordinate the relaypilot sequence and modification matrix with the special form of orthogonality, which adds additional complexity to power allocation and joint optimization.

A typical example is the AF-based diamond relay network consisting of source node (SN), destination node (DN), and two AF half-duplex relay nodes with no direct link between the source and the destination [19]. In this paper, we consider an AF-based diamond relay network and propose a modified relay-assisted training strategy to estimate second-hop link. Our strategy is to transmit the source training and data in time-multiplexing (TM) mode from the SN, while each relay superimposes its specialized relay training over the amplified received data vectors. In this manner, the complex problem of a joint optimization design of source training and relay training is disassembled into two independent optimization design problems. In the work, two methods are employed to eliminate cooperative data interference (CDI) and multiaccess interference (MAI). Firstly, to remove the effects of the unknown cooperative information-induced interference on the estimator of second-hop links, some cooperative information-bearing data tones of the received signal at each relay node are discarded to accommodate relay training sequence to keep the orthogonality between data and training. Meanwhile, a simple iterative reconstruction method is employed to compensate the distortion at the DN. Secondly, we derive a couple of optimal zero-correlation zone (ZCZ) relay-assisted sequences designed to eliminate the MAI and minimize the MSE when estimating the secondhop $\left(\mathbb{R}_{1}-\mathbb{D}, \mathbb{R}_{2}-\mathbb{D}\right)$ channel $[20,21]$. At the $\mathrm{DN}$, the received signals from each relay node are combined to achieve spatial diversity and enhance data reliability. The simulation results are presented to prove the performance of the proposed schemes.

The rest of the paper is organized as follows. In Section 2, we present the AF-based diamond relay network. The design of the training sequences at relay nodes is described in Section 3. The detection performance and iterative reconstruction method are introduced in Section 4 . The simulations results are presented in Section 5 and conclusions are drawn in Section 6.

Notation 1. Superscripts $H, T$, and $\dagger$ denote the complex conjugate transpose, transpose, and pseudo-inverse, respectively. The $N \times N$ identity matrix is denoted by $\mathbf{I}$. The Discrete Fourier Transform (DFT) of the $N \times 1$ vector $\mathbf{x}$ is denoted by $\widetilde{\mathbf{x}}=\mathbf{F}_{N} \mathbf{x}$, where $\mathbf{F}_{N}$ has $(m, n)$ entry $1 / \sqrt{N} e^{-j 2 \pi m n / N}$.

\section{System Model}

We consider a single-carrier transmission in the AF-based diamond relay network operating in a frequency-selective fading environment, where the data is transmitted from the $\mathrm{SN}$ to the DN through two relay nodes $\mathbb{R}_{1}, \mathbb{R}_{2}$ as shown in

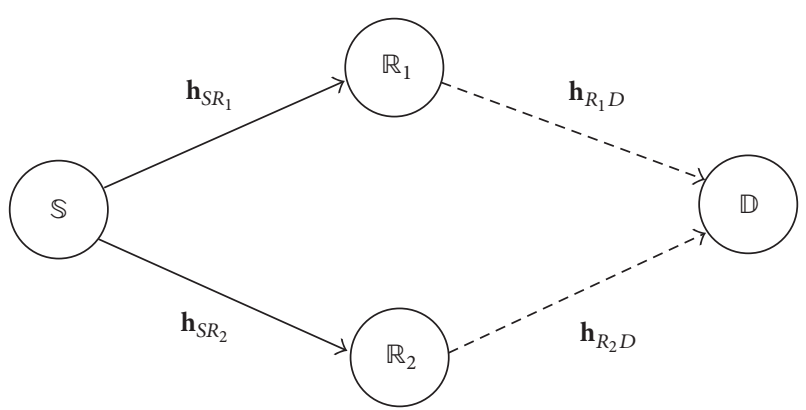

$\longrightarrow$ First slot

$--\rightarrow$ Second slot

FIGURE 1: Diagram for cooperative diversity with two relay nodes.

$\mathbb{S}$\begin{tabular}{lll}
\hline $\mathbf{t}$ & $\mathbf{d}$ \\
& $\mathbb{R}_{i} \quad \mathbf{t}^{S}$ & $\mathbf{d}$ \\
\hline & & $\mathbf{d}$ \\
\hline
\end{tabular}

FIGURE 2: Structure of one frame with relay-assisted training.

Figure 1. The individual channel impulse response links are defined as $\mathbf{h}_{S R_{i}}=\left[h_{S R_{i}}(0), h_{S R_{i}}(1), \ldots, h_{S R_{i}}\left(L_{S R_{i}}-1\right)\right]^{T}, \mathbf{h}_{R_{i} D}=$ $\left[h_{R_{i} D}(0), h_{R_{i} D}(1), \ldots, h_{R_{i} D}\left(L_{R_{i} D}-1\right)\right]^{T}, i=1$, 2. Individual channel taps are independent and Rayleigh-distributed as $h_{S R_{i}}(l) \sim \mathscr{C} \mathscr{N}\left(0, \sigma_{S R_{i}, l}^{2}\right), h_{R_{i} D}(l) \sim \mathscr{C} \mathcal{N}\left(0, \sigma_{R_{i} D, l}^{2}\right)$. The signal transmission between $\mathbb{S}$ and $\mathbb{D}$ can be partitioned into two time slots. We consider a cyclic prefix (CP) single-carrier transmission system and assume perfect synchronization for both transmission phases.

At the $\mathrm{SN}$, we first design a frame consisting of the training block $\mathbf{t}^{S}=\left[t^{S}(0), t^{S}(1), \ldots, t^{S}(N-1)\right]^{T}$ and the data block $\mathbf{d}=[d(0), d(1), \ldots, d(N-1)]^{T}$, where $N$ denotes the block length. To avoid interblock interference at both relay nodes and the DN, a CP of a length $L_{c p} \geq \max \left\{L_{S R_{1}}-1, L_{S R_{2}}-\right.$ $1\}$ is inserted into the front of each block before transmission and is removed after reception. During the first time slot, the SN transmits one data block to each relay nodes with average power of $E\left[\left(\mathbf{t}^{S}\right)^{H} \mathbf{t}^{S}\right]=E\left[\mathbf{d}^{H} \mathbf{d}\right]=N P_{s}$.

At the second time slot, $\mathbb{R}_{1}$ and $\mathbb{R}_{2}$ amplify the received signal and forward the signal to $\mathbb{D}$ with average power of $E\left[\left(\mathbf{z}_{i}^{t}\right)^{H} \mathbf{z}_{i}^{t}\right]=E\left[\left(\mathbf{z}_{i}^{d}\right)^{H} \mathbf{z}_{i}^{d}\right]=N P_{r}$, respectively. In our strategy, relay-assisted training is superimposed onto the top of the amplified data vector as illustrated in Figure 2. Due to MAI, $\mathbb{D}$ cannot identify the corresponding relay channels. Therefore, linear modification diagonal matrix is designed for training signal at each relay.

After the signal processing at each relay node, the retransmitted signal is given by, respectively,

$$
\begin{aligned}
& \mathbf{z}_{i}^{t}=\beta_{i}^{t} \boldsymbol{\Lambda}_{i} \mathbf{r}_{i}^{t}=\beta_{i}^{t} \boldsymbol{\Lambda}_{i} \mathbf{H}_{S R_{i}} \mathbf{t}^{S}+\beta_{i}^{t} \boldsymbol{\Lambda}_{i} \mathbf{n}_{R_{i}}^{t}, \\
& \mathbf{z}_{i}^{d}=\beta_{i}^{d} \mathbf{r}_{i}^{d}+\mathbf{t}^{R_{i}}=\beta_{i}^{d} \mathbf{H}_{S R_{i}} \mathbf{d}+\mathbf{t}^{R_{i}}+\beta_{i}^{d} \mathbf{n}_{R_{i}}^{d},
\end{aligned}
$$


where $\mathbf{H}_{S R_{i}}$ is an $N \times N$ circulant matrix, with the first columns $\left[\left(\mathbf{h}_{S R_{i}}\right)^{T}, \mathbf{0}_{1 \times\left(N-L_{S R_{i}}\right.}\right)^{T}$, and $\mathbf{n}_{R_{i}}^{t}, \mathbf{n}_{R_{i}}^{d}$ are circular complex white Gaussian noise with zero mean and covariance matrix $\operatorname{cov}\left(\mathbf{n}_{S R_{i}}^{t}\right)=\operatorname{cov}\left(\mathbf{n}_{S R_{i}}^{d}\right)=\sigma_{n}^{2} \mathbf{I}, \gamma P_{r_{i}}$ that is allocated to the relay training, that is, $E\left[\left(t^{R_{i}}\right)^{H} t^{R_{i}}\right]=\gamma N P_{r_{i}}$, and $(1-$ r) $P_{r_{i}}$ is allocated to the amplified received data, where $0<\gamma<1$. The modification diagonal matrix $\Lambda_{i}=$ $\operatorname{diag}\left\{\Lambda_{i}(0), \Lambda_{i}(1), \ldots, \Lambda_{i}(N-1)\right\}$ is set, where $\Lambda_{i}(k)=$ $e^{j 2 \pi(i-1)(k-1) / N}$ and the amplified factors are given by

$$
\begin{aligned}
& \beta_{i}^{t}=\sqrt{\frac{P_{r_{i}}}{P_{s} \sigma_{h_{S R_{i}}^{2}}^{2}+\sigma_{n}^{2}}}, \\
& \beta_{i}^{d}=\sqrt{\frac{(1-\gamma) P_{r_{i}}}{P_{s} \sigma_{h_{S R_{i}}^{2}}^{2}+\sigma_{n}^{2}}},
\end{aligned}
$$

where $\sigma_{h_{S R_{i}}}^{2}=\sum_{l=0}^{L_{S R_{i}}-1} \sigma_{h_{S R_{i}},}^{2}$. The received signal at the DN can be expressed by

$$
\begin{aligned}
\mathbf{y}^{t} & =\sum_{i=1}^{2} \mathbf{H}_{R_{i} D} \beta_{i}^{t} \boldsymbol{\Lambda}_{i} \mathbf{r}_{i}^{t}+\mathbf{n}_{D}^{t} \\
& =\beta_{1}^{t} \mathbf{H}_{R_{1} D} \boldsymbol{\Lambda}_{1} \mathbf{H}_{S R_{1}} \mathbf{t}^{S}+\beta_{2}^{t} \mathbf{H}_{R_{2} D} \boldsymbol{\Lambda}_{2} \mathbf{H}_{S R_{2}} \mathbf{t}^{S}+\mathbf{n}_{Z}^{t}, \\
\mathbf{y}^{d} & =\sum_{i=1}^{2} \mathbf{H}_{R_{i} D} \beta_{i}^{d} \mathbf{r}_{i}^{d}+\mathbf{n}_{D}^{d} \\
& =\left(\beta_{1}^{d} \mathbf{H}_{R_{1}}+\beta_{2}^{d} \mathbf{H}_{R_{2}}\right) \mathbf{d}+\mathbf{H}_{R_{1} D} \mathbf{t}^{R_{1}}+\mathbf{H}_{R_{2} D} \mathbf{t}^{R_{2}}+\mathbf{n}_{Z}^{d},
\end{aligned}
$$

where $\mathbf{H}_{R_{i} D}$ is an $N \times N$ circulant matrix, with the first columns $\left[\left(\mathbf{h}_{R_{i} D}\right)^{T}, \mathbf{0}_{1 \times\left(N-L_{R_{i} D}\right)}\right]^{T}, \mathbf{n}_{D}^{t}, \mathbf{n}_{D}^{d}$ are circular complex white Gaussian noise, $\mathbf{H}_{R_{i}}=\mathbf{H}_{S R_{i}} \mathbf{H}_{R_{i} D}$, with $L_{R_{i}}=L_{S R_{i}}+$ $L_{R_{i} D}-1, L_{S}=\max \left\{L_{R_{1}}, L_{R_{2}}\right\}$. And $\mathbf{n}_{Z}^{t}=\sum_{i=1}^{2} \beta_{i}^{t} \Lambda_{i} \mathbf{n}_{R_{i}}^{t}+\mathbf{n}_{D}^{t}$, $\mathbf{n}_{Z}^{d}=\sum_{i=1}^{2} \beta_{i}^{d} \mathbf{n}_{R_{i}}^{d}+\mathbf{n}_{D}^{d}, \mathbf{n}_{R_{i}}^{t}, \mathbf{n}_{R_{i}}^{d}$ are also circular complex white Gaussian noise.

\section{Design of the Relay Training Sequence}

With the purpose of signal retrieving and obtaining diversity, individual channel estimation is required in a diamond relay network. From (4), the received data signal can be expressed as follows:

$$
\begin{aligned}
\mathbf{y}^{d} & =\boldsymbol{\Phi}_{L_{R_{1} D}}\left[\mathbf{t}^{R_{1}}\right] \mathbf{h}_{R_{1} D} \\
& +\underbrace{\Phi_{L_{S}}[\mathbf{d}]\left(\beta_{1}^{d} \mathbf{h}_{R_{1}}+\beta_{2}^{d} \mathbf{h}_{R_{2}}\right)+\boldsymbol{\Phi}_{L_{R_{2} D}}\left[\mathbf{t}^{R_{2}}\right] \mathbf{h}_{R_{2} D}+\mathbf{n}_{Z}^{d}}_{\mathbf{v}_{d}},
\end{aligned}
$$

where $\boldsymbol{\Phi}_{L}[\mathbf{x}]$ is an $N \times L$ column-wise circulant matrix with the first column $\mathbf{x}$. It is noted that $\mathbf{v}_{d}$ includes the equivalent noise $\mathbf{n}_{Z}^{d}$ that is related to the specific realization of $\mathbf{h}_{R_{i} D}$, the extra CDI term $\boldsymbol{\Phi}_{L_{S}}[\mathbf{d}]\left(\beta_{1}^{d} \mathbf{h}_{R_{1}}+\beta_{2}^{d} \mathbf{h}_{R_{2}}\right)$, and the MAI term $\boldsymbol{\Phi}_{L_{R_{2} D}}\left[\mathbf{t}^{R_{2}}\right] \mathbf{h}_{R_{2} D}$. Assuming that the received data, training sequence, and noise in (5) are mutually independent, the covariance matrices of $\mathbf{v}_{d}$ can be written, respectively, as

$$
\begin{aligned}
\mathbf{C}_{d} & =E\left(\mathbf{v}_{d} \mathbf{v}_{d}^{H}\right)=\left[\left(\beta_{1}^{d} \sigma_{h_{R_{1}}}^{2}+\beta_{2}^{d} \sigma_{h_{R_{2}}}^{2}\right) P_{s}+\sigma_{h_{R_{2} D}}^{2} \gamma P_{r_{2}}\right. \\
& \left.+\left(\beta_{1}^{d} \sigma_{h_{R_{1} D}}^{2}+\beta_{2}^{d} \sigma_{h_{R_{2} D}}^{2}+1\right) \sigma_{n}^{2}\right] \mathbf{I}_{N}=\sigma_{v_{b}}^{2} \mathbf{I}_{N},
\end{aligned}
$$

where $\sigma_{h_{R_{i} D}}^{2}=\sum_{l=0}^{L_{R_{i} D}-1} \sigma_{h_{R_{i} D},}^{2}, \sigma_{h_{R_{i}}}^{2}=\sum_{l=0}^{L_{S R_{i}}+L_{R_{i} D}-2} \sigma_{h_{R_{i}, l}}^{2}$ with $\sigma_{h_{R_{i}, l}}^{2}=\sum_{j=0}^{l} \sigma_{h_{S R_{i}, j}}^{2} \sigma_{h_{R_{i} \mathrm{D},(l-j)}}^{2}$. Without loss of generality, we take the estimation of $\widehat{\mathbf{h}}_{R_{1} D}$ as an example, and the traditional Least Square (LS) estimation of the second-hop channel is obtained by

$$
\begin{aligned}
& \widehat{\mathbf{h}}_{R_{1} D}=\boldsymbol{\Phi}_{L_{R_{1} D}}^{\dagger}\left[\mathbf{t}^{R_{1}}\right] \mathbf{y}^{d}=\mathbf{h}_{R_{1} D}+\boldsymbol{\Phi}_{L_{R_{1} D}}^{\dagger}\left[\mathbf{t}^{R_{1}}\right] \\
& \cdot\left\{\boldsymbol{\Phi}_{L_{S}}[\mathbf{d}]\left(\beta_{1}^{d} \mathbf{h}_{R_{1}}+\beta_{2}^{d} \mathbf{h}_{R_{2}}\right)+\boldsymbol{\Phi}_{L_{R_{2} D}}\left[\mathbf{t}^{R_{2}}\right] \mathbf{h}_{R_{2} D}\right. \\
& \left.+\mathbf{n}_{Z}^{d}\right\} .
\end{aligned}
$$

The MSE of the LS estimator can be given by

$$
\begin{aligned}
\operatorname{MSE}_{h_{R_{1} D}} & =E\left\{\left\|\widehat{\mathbf{h}}_{R_{1} D}-\mathbf{h}_{R_{1} D}\right\|\right\} \\
& =\operatorname{tr}\left(\boldsymbol{\Phi}_{L_{R_{1} D}}^{\dagger}\left[\mathbf{t}^{R_{1}}\right] \mathbf{C}_{d} \boldsymbol{\Phi}_{L_{R_{1} D}}^{\dagger H}\left[\mathbf{t}^{R_{1}}\right]\right) \\
& =\sigma_{v_{d}}^{2} \operatorname{tr}\left[\left(\boldsymbol{\Phi}_{L_{R_{1} D}}^{H}\left[\mathbf{t}^{R_{1}}\right] \boldsymbol{\Phi}_{L_{R_{1} D}}\left[\mathbf{t}^{R_{1}}\right]\right)^{-1}\right] .
\end{aligned}
$$

For an $N \times N$ positive definite matrix $\mathbf{M}$, we have $\operatorname{tr}(\mathbf{M}) \operatorname{tr}\left(\mathbf{M}^{-1}\right) \geq N^{2}$, where the equality holds if and only if $\mathbf{M}=\lambda \mathbf{I}_{N}$ for some nonzero constant $\lambda$. Using this and the fact that the matrix $\left(\boldsymbol{\Phi}_{L_{R_{1} D}^{H}}^{H}\left[\mathbf{t}^{R_{1}}\right] \boldsymbol{\Phi}_{L_{R_{1} D}}\left[\mathbf{t}^{R_{1}}\right]\right)^{-1}$ is positive definite, the optimal training for a fixed power $\gamma P_{r_{1}}$ must satisfy the following conditions:

$$
\text { C1: } \boldsymbol{\Phi}_{L_{R_{i} D}}^{H}\left[\mathbf{t}^{R_{i}}\right] \boldsymbol{\Phi}_{L_{R_{i} D}}\left[\mathbf{t}^{R_{i}}\right]=\gamma N P_{r_{i}} \mathbf{I}_{L_{R_{i} D}}
$$

and the Minimum Mean-Square Error (MSE) of the LS estimation is given by

$$
\operatorname{MSE}_{h_{R_{1} D}}=\frac{\sigma_{v_{b}}^{2} L_{R_{i} D}^{2}}{\operatorname{tr}\left(\boldsymbol{\Phi}_{L_{R_{1} D}}^{H}\left[\mathbf{t}^{R_{1}}\right] \boldsymbol{\Phi}_{L_{R_{1} D}}\left[\mathbf{t}^{R_{1}}\right]\right)}=\frac{L_{R_{1} D}\left[\left(\beta_{1}^{d} \sigma_{h_{R_{1}}}^{2}+\beta_{2}^{d} \sigma_{h_{R_{2}}}^{2}\right) P_{s}+\sigma_{h_{R_{2}} D}^{2} \gamma P_{r_{2}}+\left(\beta_{1}^{d} \sigma_{h_{R_{1} D}}^{2}+\beta_{2}^{d} \sigma_{h_{R_{2} D}}^{2}+1\right) \sigma_{n}^{2}\right]}{N \gamma P_{r_{1}}} .
$$




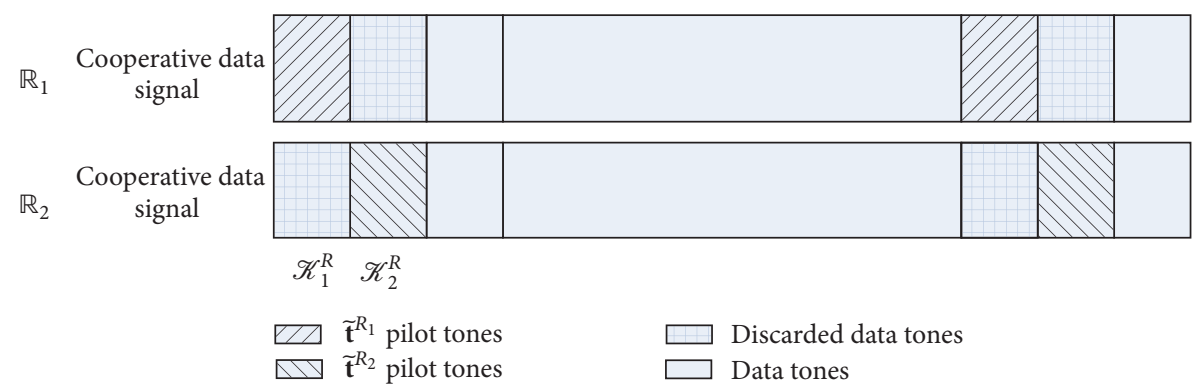

FIGURE 3: Sketch of the structure in the frequency domain for Scheme A.

The MSE of the second-hop channel in (10) consists of three terms. The first term is related to the CDI, the second term is related to the MAI, and the third term is related to the equivalent noise, all of which have serious effects on the estimate of the MSE.

(A) Cooperative Data Interference and Relay-Propagated Noise Cancellation. To eliminate the effects of the unknown CDI and relay-propagated noise on the estimator of the secondhop links, it is crucial to perform signal preprocessing at each relay node. Because relay-assisted training is periodic and its energy is concentrated only at the equispaced frequency pins, we discard some cooperative data tones of the received signal at each relay node so that the DFT at the specific frequency pins is identically zero. In this way, we construct an orthogonal structure between the cooperative data and the relay-assisted training. In fact, the discarded cooperative data tones include both the CDI and the relay-introduced noise terms. At each relay node, we subtract the vector $\mathbf{e}=\mathbf{J}_{R} \mathbf{r}_{i}^{d}$ from the received signal $\mathbf{r}_{i}^{d}$, where $\mathbf{J}_{R}=\mathbf{F}^{H} \widetilde{\mathbf{J}}_{R} \mathbf{F}$. Here, we define an $N \times N$ diagonal matrix in the frequency domain $\widetilde{\mathbf{J}}_{R}=\operatorname{diag}\left\{\widetilde{J}_{R}(0), \widetilde{J}_{R}(1), \ldots, \widetilde{J}_{R}(N-1)\right\}$ with

$$
\widetilde{J}_{R}(k)=\left\{\begin{array}{ll}
1 & k \in \mathscr{K}_{1}^{R} \\
0 & k \notin \mathscr{K}_{1}^{R}
\end{array} .\right.
$$

Assume that the indices of the nonzero pilot tones corresponding to $\mathbf{t}^{R_{1}}$ and $\mathbf{t}^{R_{2}}$ belong to $\mathscr{K}_{1}^{R}$. The data signal at $\mathbb{R}_{i}$ can be refreshed as follows:

$$
\begin{aligned}
\mathbf{z}_{i}^{d} & =\beta_{i}^{d}\left(\mathbf{r}_{i}^{d}-\mathbf{e}\right)+\mathbf{t}^{R_{i}} \\
& =\beta_{i}^{d}\left(\mathbf{I}-\mathbf{J}_{R}\right) \mathbf{H}_{S R_{i}} \mathbf{d}+\beta_{i}^{d}\left(\mathbf{I}-\mathbf{J}_{R}\right) \mathbf{n}_{S R_{i}}^{d}+\mathbf{t}^{R_{i}} \\
& =\beta_{i}^{d}\left(\mathbf{H}_{S R_{i}} \mathbf{d}+\mathbf{n}_{S R_{i}}^{d}\right)-\beta_{i}^{d} \mathbf{J}_{R}\left(\mathbf{H}_{S R_{i}} \mathbf{d}+\mathbf{n}_{S R_{i}}^{d}\right)+\mathbf{t}^{R_{i}} .
\end{aligned}
$$

(B) Multiaccess Interference Cancellation. With the aim of minimizing the MSE of second-hop channel, the following constrained conditions $\mathbf{C}_{2}$ and $\mathbf{C}_{3}$ should be satisfied according to (7):

$$
\begin{aligned}
& \mathbf{C}_{2}: \boldsymbol{\Phi}_{L_{R_{1} D}}^{H}\left[\mathbf{t}^{R_{1}}\right] \boldsymbol{\Phi}_{L_{R_{2} D}}\left[\mathbf{t}^{R_{2}}\right]=\mathbf{0}, \\
& \mathbf{C}_{3}: \boldsymbol{\Phi}_{L_{R_{2} D}}^{H}\left[\mathbf{t}^{R_{2}}\right] \boldsymbol{\Phi}_{L_{R_{1} D}}\left[\mathbf{t}^{R_{1}}\right]=\mathbf{0}
\end{aligned}
$$

In Scheme $A$, a couple of relay training sequences $\left(t^{R_{1}}\right.$ and $\mathbf{t}^{R_{2}}$ ) with their energy concentrated at different frequency pins is designed. We assume that the indices of the nonzero pilot tones corresponding to $\mathbf{t}^{R_{1}}$ belong to $\mathscr{K}_{1}^{R}$, while the indices of the nonzero pilot tones corresponding to $\mathbf{t}^{R_{2}}$ belong to $\mathscr{K}_{2}^{R}$. The condition $\mathscr{K}_{1}^{R} \cap \mathscr{K}_{2}^{R}=\varnothing$ should be satisfied. With pilot tones occupying the disjoint frequency tones, the problem of MAI can be solved. However, it is necessary to perform signal preprocessing at each relay node. As a result, to maintain the orthogonality, it is indispensable that the frequency components of the received signal corresponding to both $\mathscr{K}_{1}^{R}$ and $\mathscr{K}_{2}^{R}$ should be removed to accommodate relaypilot tones as illustrated in Figure 3. Clearly, the distortion of the received data would be doubled in Scheme A and the detection performance will be seriously degraded. Therefore, this is not an appropriate scheme to avoid MAI.

In Scheme B, a couple of relay training with autocorrelation and cross-correlation properties are designed. In the ZCZ sequence group $\left\{t^{R_{1}}(k), t^{R_{2}}(k)\right\}, P$ denotes the period of the ZCZ sequence, $Z$ denotes the length of the zerocorrelation zone, and the condition $Z \geq \max \left\{L_{R_{1} D^{-}}-1, L_{R_{2} D^{-}}\right.$ 1 ) is required, where $N=P Q$ and $Q$ is an integer. The autocorrelation and cross-correlation properties of the ZCZ sequence are stated as follows:

$$
\begin{aligned}
R_{i, j}(\tau)= & \sum_{k=0}^{P-1} t^{R_{i}}(k) t^{R_{j}}(k+\tau) \\
= & \left\{\begin{array}{l}
P, \text { if } \tau=0, i=j \\
0, \text { if } 0<\tau \leq Z, i=j \\
0, \text { if } 0 \leq \tau \leq Z, i \neq j,
\end{array}\right.
\end{aligned}
$$

where $i, j=1,2$. The relay training sequences can satisfy the conditions $\mathbf{C}_{2}$ and $\mathbf{C}_{3}$ denoted in (13). Moreover, the indices of the nonzero pilot tones corresponding to the $\mathrm{ZCZ}$ sequence group including $\mathbf{t}^{R_{1}}$ and $\mathbf{t}^{R_{2}}$ both belong to $\mathscr{K}_{1}^{R}$. As a result, only the frequency components corresponding to $\mathscr{K}_{1}^{R}$ should be removed. This is the optimal scheme to avoid MAI. The structure of the data block in the frequency domain is illustrated in Figure 4.

At the DN, the decoupling of the two relay training sequences is easily realized. In essence, the complicated twoaccess channel estimation problem is decomposed into two 


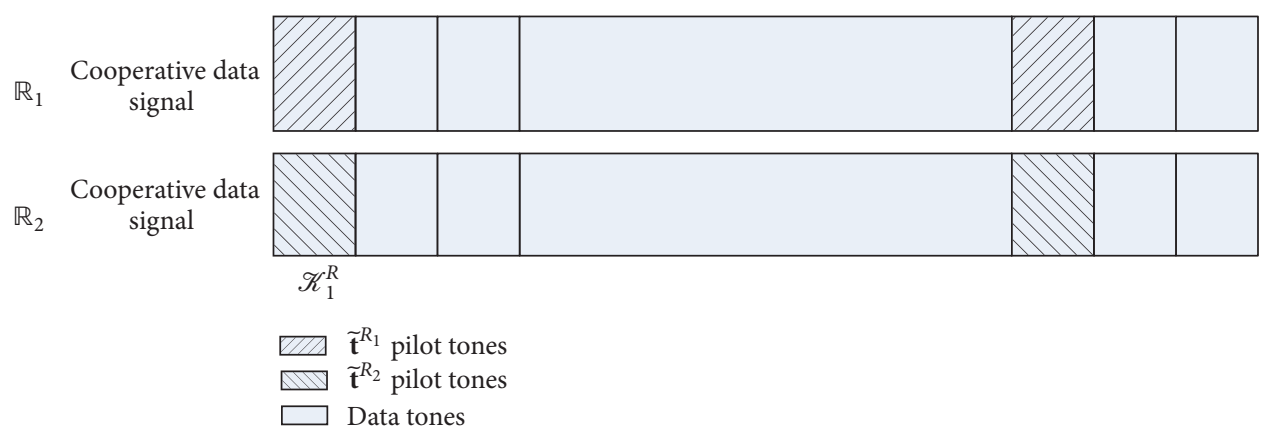

FIGURE 4: Sketch of the structure in the frequency domain for Scheme B.

independent channel estimation problems. According to (5) and (12), the received data signal at the DN can be refreshed as follows:

$$
\begin{aligned}
\mathbf{y}^{d}= & \boldsymbol{\Phi}_{L_{R_{1} D}}\left[\mathbf{t}^{R_{1}}\right] \mathbf{h}_{R_{1} D} \\
& +\boldsymbol{\Phi}_{L_{S}}\left[\left(\mathbf{I}-\mathbf{J}_{R}\right) \mathbf{d}\right]\left(\beta_{1}^{d} \mathbf{h}_{R_{1}}+\beta_{2}^{d} \mathbf{h}_{R_{2}}\right) \\
& +\boldsymbol{\Phi}_{L_{R_{2} D}}\left[\mathbf{t}^{R_{2}}\right] \mathbf{h}_{R_{2} D}+\overline{\mathbf{n}}_{Z}^{d},
\end{aligned}
$$

where $\overline{\mathbf{n}}_{Z}^{d}=\beta_{1}^{d} \boldsymbol{\Phi}_{L_{R_{1} D}}\left[\left(\mathbf{I}-\mathbf{J}_{R}\right) \mathbf{n}_{S R_{1}}^{d}\right] \mathbf{h}_{R_{1} D}+\beta_{2}^{d} \boldsymbol{\Phi}_{L_{R_{2} D}}[(\mathbf{I}-$ $\left.\left.\mathbf{J}_{R}\right) \mathbf{n}_{S R_{2}}^{d}\right] \mathbf{h}_{R_{2} D}+\mathbf{n}_{R D}^{d}$. It is noted that the partial cooperative data vector [the second term in (15)] and the relay training vector [the third term in (15)] occupy the disjoint frequency tones, respectively. As a result, the cooperative data have no effect on the estimation of the second-hop channel. According to C1, the pseudo-inverse of $\boldsymbol{\Phi}_{L_{R_{1} D}}\left[\mathbf{t}^{R_{1}}\right]$ can be denoted as

$$
\begin{aligned}
& \boldsymbol{\Phi}_{L_{R_{1} D}}^{\dagger}\left[\mathbf{t}^{R_{1}}\right] \\
& =\left(\boldsymbol{\Phi}_{L_{R_{1} D}}^{H}\left[\mathbf{t}^{R_{1}}\right] \boldsymbol{\Phi}_{L_{R_{1} D}}\left[\mathbf{t}^{R_{1}}\right]\right)^{-1} \boldsymbol{\Phi}_{L_{R_{1} D}}^{H}\left[\mathbf{t}^{R_{1}}\right] \\
& =\frac{1}{\gamma N P_{r_{1}}} \boldsymbol{\Phi}_{L_{R_{1} D}}^{H}\left[\mathbf{t}^{R_{1}}\right] .
\end{aligned}
$$

In the LS estimation, according to $\mathbf{C}_{2}$ and $\mathbf{C}_{3}$, the improved estimation of the second-hop channel is obtained by

$$
\begin{gathered}
\widehat{\mathbf{h}}_{R_{1} D}=\Phi_{L_{R_{1} D}}^{\dagger}\left[\mathbf{t}^{R_{1}}\right] \mathbf{y}^{d}=\mathbf{h}_{R_{1} D}+\frac{1}{\gamma N P_{r_{1}}} \boldsymbol{\Phi}_{L_{R_{1} D}}^{H}\left[\mathbf{t}^{R_{1}}\right] \\
\cdot\left\{\boldsymbol{\Phi}_{L_{S}}\left[\left(\mathbf{I}-\mathbf{J}_{R}\right) \mathbf{d}\right]\left(\beta_{1}^{d} \mathbf{h}_{R_{1}}+\beta_{2}^{d} \mathbf{h}_{R_{2}}\right)\right. \\
\left.+\boldsymbol{\Phi}_{L_{R_{2} D}}\left[\mathbf{t}^{R_{2}}\right] \mathbf{h}_{R_{2} D}+\overline{\mathbf{n}}_{Z}^{d}\right\}=\mathbf{h}_{R_{1} D}+\frac{1}{\gamma N P_{r_{1}}} \\
\cdot \boldsymbol{\Phi}_{L_{R_{1} D}^{H}}^{H}\left[\mathbf{t}^{R_{1}}\right] \mathbf{n}_{R D^{.}}^{d}
\end{gathered}
$$

According to (17), the relay-propagated noise introduced from each relay node is removed thoroughly. As both the CDI and MAI are removed, the minimum MSE of the improved LS estimation is given by

$$
\operatorname{MSE}_{h_{R_{1} D}}=\frac{L_{R_{1} D} \sigma_{n}^{2}}{\gamma N P_{r_{1}}} .
$$

\section{Diversity Combining and Iterative Reconstruction}

With the purpose of signal retrieving and obtaining diversity, individual channel $\left(\mathbb{S}-\mathbb{R}_{1}\right.$ and $\left.\mathbb{S}-\mathbb{R}_{2}\right)$ estimation is required in diamond relay networks. With the estimated second-hop channels $\widehat{\mathbf{h}}_{R_{1} D}$ and $\widehat{\mathbf{h}}_{R_{2} D}$, we employ the LS channel estimation in [12] to obtain estimated channels $\widehat{\mathbf{h}}_{S R_{1}}$ and $\widehat{\mathbf{h}}_{S R_{2}}$. After removing the contribution of the relay training sequences from $\mathbf{y}^{d}$ by simply computing $\mathbf{y}_{r e}^{d}=\left(\mathbf{I}-\mathbf{J}_{R}\right) \mathbf{y}^{d}$, the DFT of $\mathbf{y}_{\mathrm{re}}^{d}$ can be written as

$$
\begin{aligned}
\widetilde{\mathbf{y}}_{\mathrm{re}}^{d}= & \left(\beta_{1}^{d} \widetilde{\mathbf{H}}_{R_{1} D} \widetilde{\mathbf{H}}_{S R_{1}}+\beta_{1}^{d} \widetilde{\mathbf{H}}_{R_{2} D} \widetilde{\mathbf{H}}_{S R_{2}}\right)\left(\mathbf{I}-\widetilde{\mathbf{J}}_{R}\right) \tilde{\mathbf{d}} \\
& +\left(\mathbf{I}-\widetilde{\mathbf{J}}_{R}\right) \widetilde{\mathbf{n}}_{Z}^{d} .
\end{aligned}
$$

The equalized signal is given by

$$
\widetilde{\mathbf{u}}=\boldsymbol{\Sigma}_{S}^{H} \mathbf{R}_{\mathbf{y}}^{-1} \widetilde{\mathbf{y}}_{\mathrm{re}},
$$

where $\Sigma_{S}=\beta_{1}^{d} \widehat{\widetilde{\mathbf{H}}}_{S R_{1}} \widehat{\widetilde{\mathbf{H}}}_{R_{1} D}+\beta_{2}^{d} \widehat{\widetilde{\mathbf{H}}}_{S R_{2}} \widehat{\widetilde{\mathbf{H}}}_{R_{2} D}, \mathbf{R}_{\mathbf{y}}=\boldsymbol{\Sigma}_{S} \boldsymbol{\Sigma}_{S}^{H}+$ $\left[\left(\beta_{1}^{d}\right)^{2} \widehat{\widetilde{\mathbf{H}}}_{R_{1} D} \widehat{\widetilde{\mathbf{H}}}_{R_{1} D}^{H}+\left(\beta_{2}^{d}\right)^{2} \widehat{\widetilde{\mathbf{H}}}_{R_{2} D} \widehat{\widetilde{\mathbf{H}}}_{R_{2} D}^{H}+\mathbf{I}\right] \widehat{\sigma}_{n}^{2}$ in the case of Minimum Mean-Square Error (MMSE) equalization, $\widehat{\widehat{\mathbf{H}}}_{S R_{i}}$, $\widehat{\widetilde{\mathbf{H}}}_{R_{i} D}$ are the DFT of the estimated channels $\widehat{\mathbf{h}}_{S R_{i}}$ and $\widehat{\mathbf{h}}_{R_{1} D}$, $\widetilde{\mathbf{n}}_{Z}^{d}$ is the DFT of equivalent noise $\mathbf{n}_{Z}^{d}$, and $\widetilde{\sigma}_{n}^{2}=(1-$ $1 / Q)\left(\beta_{1}^{d} \sigma_{h_{R_{1} D}}^{2}+\beta_{2}^{d} \sigma_{h_{R_{2} D}}^{2}+1\right) \sigma_{n}^{2}$. Because the data distortion is singular, $\mathbf{d}$ cannot be recovered linearly. As a result, we employ the symbol-to-symbol iterative detection scheme. The initial hard detector of $\mathbf{d}$ is given by

$$
\widehat{\mathbf{d}}^{(0)}=\left\lfloor\mathbf{F}^{H} \widetilde{\mathbf{u}}\right\rfloor,
$$

where $\lfloor\cdot\rfloor$ stands for the decision function. For subsequent iterations, the detected symbols are utilized to compute $\mathbf{J}_{R} \widehat{\mathbf{d}}$ 
TABLE 1: Binary ZCZ sequence group (0 for -1$)$.

\begin{tabular}{|c|c|c|}
\hline$(P, Z)$ & Relay training & ZCZ sequences \\
\hline \multirow{2}{*}{$(32,4)$} & $\mathbf{t}^{R_{1}}$ & $\{11011101000100010010110111100001\}$ \\
\hline & $\mathbf{t}^{R_{2}}$ & $\{00101101111000011101110100010001\}$ \\
\hline
\end{tabular}

TABLE 2: Various system parameters for different choices.

\begin{tabular}{cccccc}
\hline & Frame $N_{f}$ & Training $N_{c}$ & Data $N_{b}$ & $\alpha=N_{c} / N_{f}$ & $\begin{array}{c}\text { BER at } \\
20 \mathrm{~dB}\end{array}$ \\
\hline Case 1 & 496 & 48 & 448 & 0.1 & 0.003768 \\
Case 2 & 640 & 64 & 576 & 0.1 & 0.00329 \\
\hline
\end{tabular}

and compensate for the data loss. The detected symbols at the next iteration are given by

$$
\widehat{\mathbf{d}}^{(i)}=\left\lfloor\mathbf{F}^{H} \widetilde{\mathbf{u}}+\mathbf{J}_{R} \widehat{\mathbf{d}}^{(i-1)}\right\rfloor .
$$

\section{Simulation Results}

In this section, we present the simulation results to evaluate performance of both the proposed scheme (denoted as "proposed" in figures) and the pilot-based scheme in [15] (denoted as "pilot-based scheme" in figures) in terms of bit error rate (BER) and normalized MSE of channel estimation. The channel is randomly generated and assumed to be uncorrelated Rayleigh fading with a length of $L_{S R_{1}}=$ $L_{S R_{2}}=L_{R_{1} D}=L_{R_{2} D}=4$. For ease of description, the block lengths are the same in this context. However, different lengths can be used based on the requirements. The equispaced and equipowered pilots are selected. The data symbols are extracted from the quadrature phase-shift keying constellation. The relay training is designed using $P=32, Z=$ 4 and the relay training sequences are set as shown in Table 1 according to $[20,21]$. SNR of each link are assumed that $\mathrm{SNR}=P_{S} / \sigma_{n}^{2}=P_{R_{1}} / \sigma_{n}^{2}=P_{R_{2}} / \sigma_{n}^{2}$, where $P_{S}=P_{R_{1}}=P_{R_{2}}=1$. In the simulation, we employ various system parameters of frame (Case 1 and Case 2), respectively, as shown in Table 2.

To obtain the optimal design for the relay training sequences, we display the bit error rate (BER) performance versus $\gamma$ for the proposed scheme under different signal-tonoise ratio (SNR) conditions in Figure 5. Clearly, the BER performance improves when $\gamma$ increases. This occurs because more power is allocated to the superimposed relay training, while less power is allocated to the data sequence. It is evident that the optimal $\gamma$ remains at the minimum value when the SNR is 10, 15, and 20, respectively. As a result, we adopt $\gamma=0.1$ as the approximately optimal value.

Figure 6 shows the MSE performance of channel estimation with different schemes for Case 1. The estimation performance of the $\mathbb{S}-\mathbb{R}_{i}$ link is worse than that of the $\mathbb{R}_{i}-\mathbb{D}$ link because the former link is affected by both the propagated noise introduced from the relay nodes and the estimation error of the second-hop link. Moreover, the performance
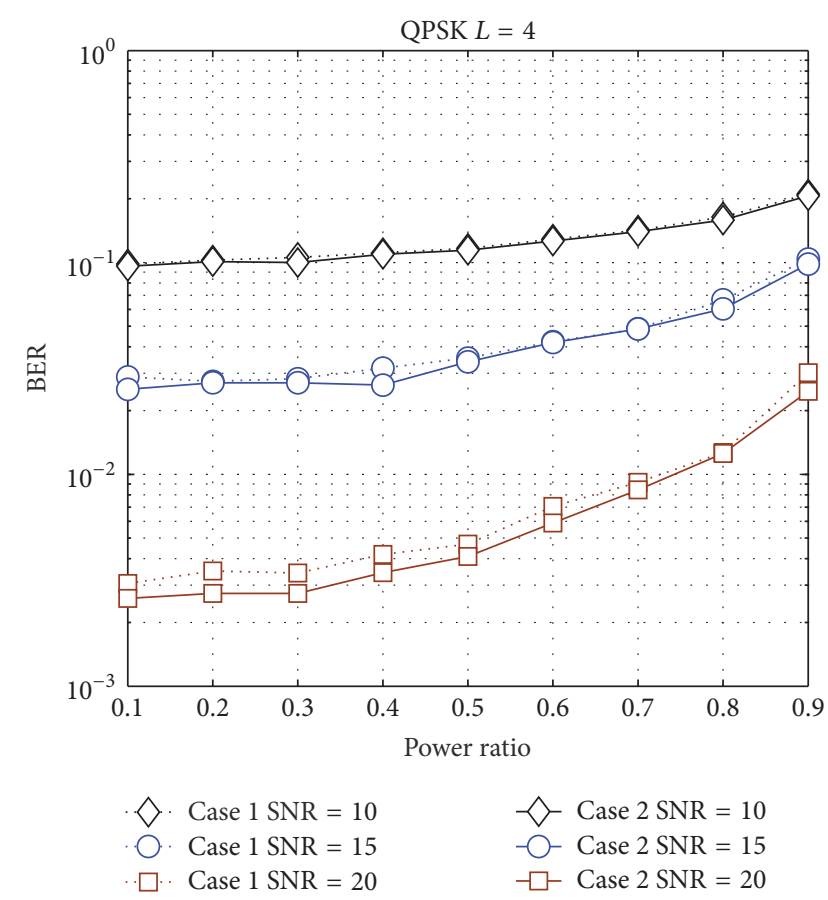

FIGURE 5: BER versus $\gamma$ for different SNR conditions.

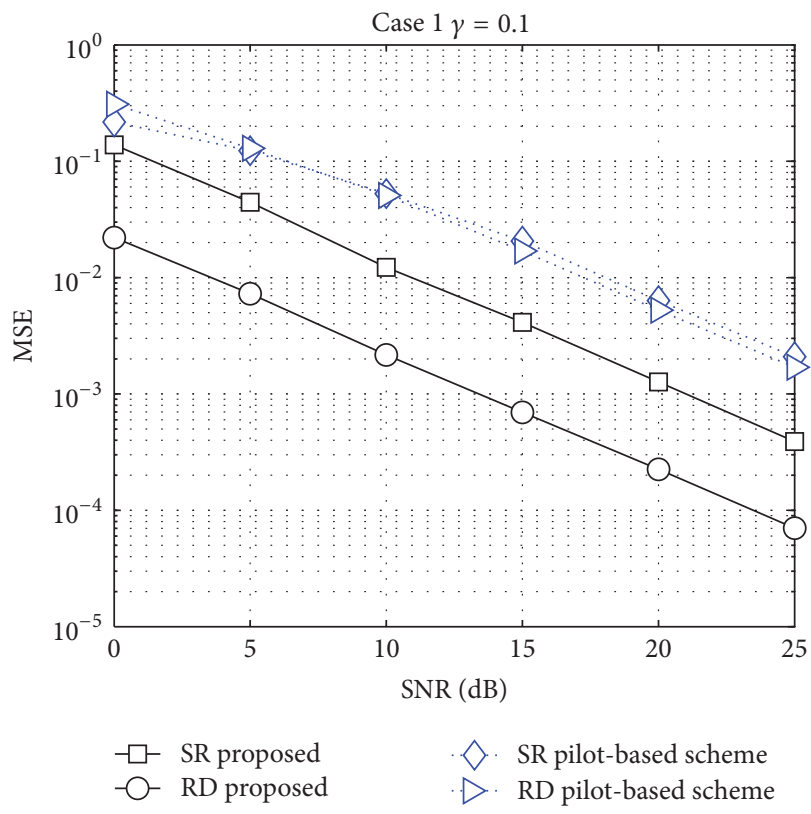

FIgURE 6: MSE performance with different schemes for Case 1. 


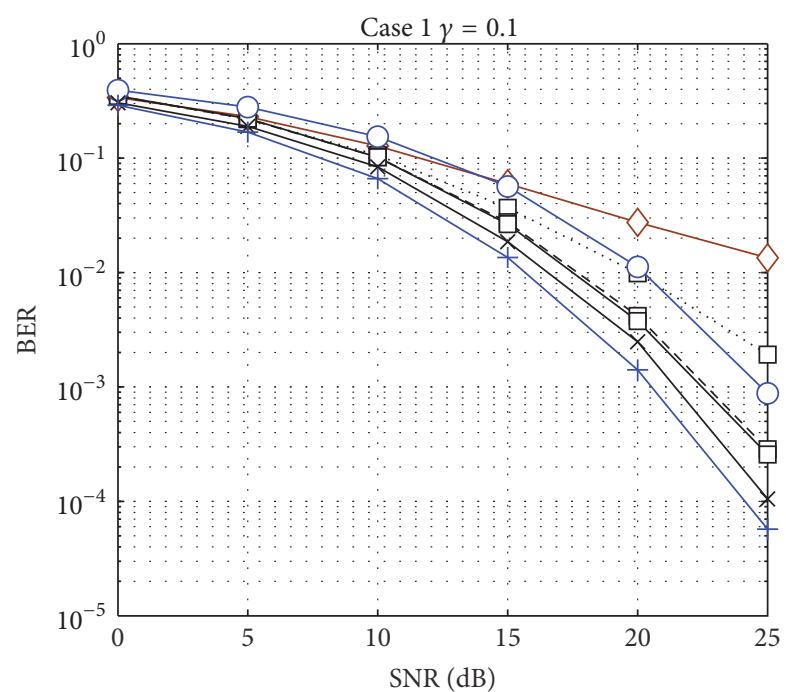

$$
\begin{aligned}
& \checkmark \text { Scheme A proposed } \quad-\bigcirc-\text { Pilot-based scheme } \\
& \text { - } \square \text {. Scheme B iter } 0 \text { proposed } \rtimes \text { Perfect CSI with } \\
& -\square \text { - Scheme B Iter } 1 \text { proposed distortion } \\
& -\square-\text { Scheme B Iter } 2 \text { proposed } \rightarrow \text { Perfect CSI without } \\
& \text { distortion }
\end{aligned}
$$

FIGURE 7: BER performance with different schemes for Case 1.

of the proposed scheme is superior to that of the pilotbased scheme described in [17]. The pilot-based scheme aims to superimpose the relay-assisted training onto the source training signal with a special orthogonal constraint. With the same average power and different block length, the total energy of the relay-assisted training in the scheme [17] is reduced compared to the proposed scheme. Moreover, the relay-propagated noise has a serious impact on the estimation of the $\mathbb{R}_{i}-\mathbb{D}$ link in the scheme [17]. As a result, the proposed estimation scheme is superior to the scheme [17].

Figure 7 shows the BER performance with different schemes for Case 1. In the proposed Scheme B, the performance improvement via the first iteration is distinct but negligible with additional iterations. It can be observed that the BER performance of the Scheme B is much better than that of Scheme A and for the scheme described in [17]. It is noted that a double distortion is induced in Scheme A and the inferior channel estimation of the pilot-based scheme clearly results in a degradation in BER. It can be predicted that a symbol error floor will occur in both Scheme A and the scheme described in [17] with an increasing SNR. After two iterations, the detection performance in Scheme B can approach that with perfect CSI, which means the channel estimation satisfies the need of the cooperative system. The performance reduction caused by the distortion can be judged in terms of the BER loss in the figure.

Figures 8 and 9 show the MSE performance and BER performance with different schemes for Case 2 . We obviously observe that the estimation performance is almost the same for Case 1 and Case 2. Moreover, the BER performance for the pilot-based scheme would remain the same with various system parameters of frame. However, with the length of data

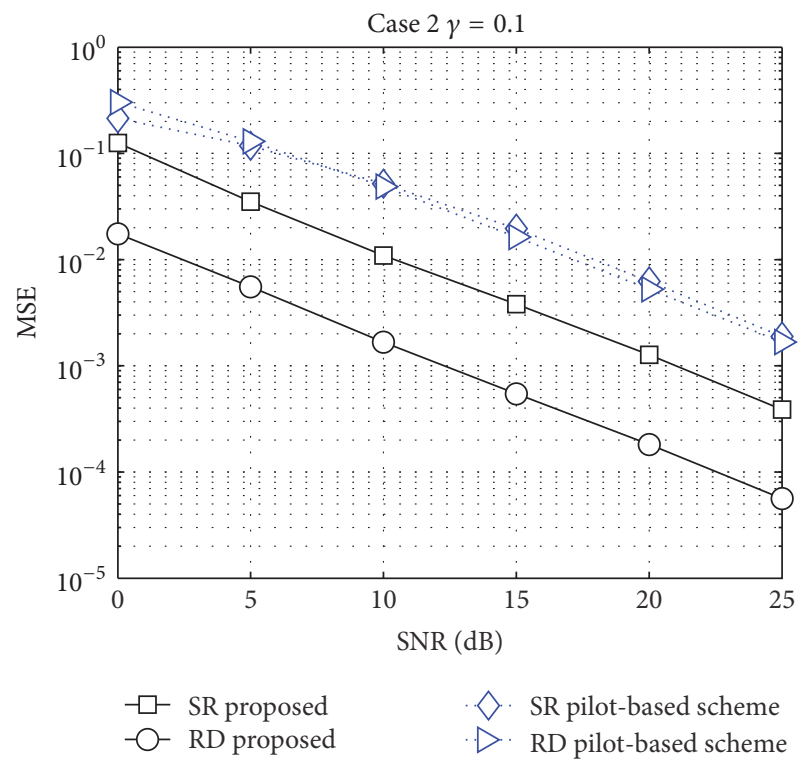

FIGURE 8: MSE performance with different schemes for Case 2.

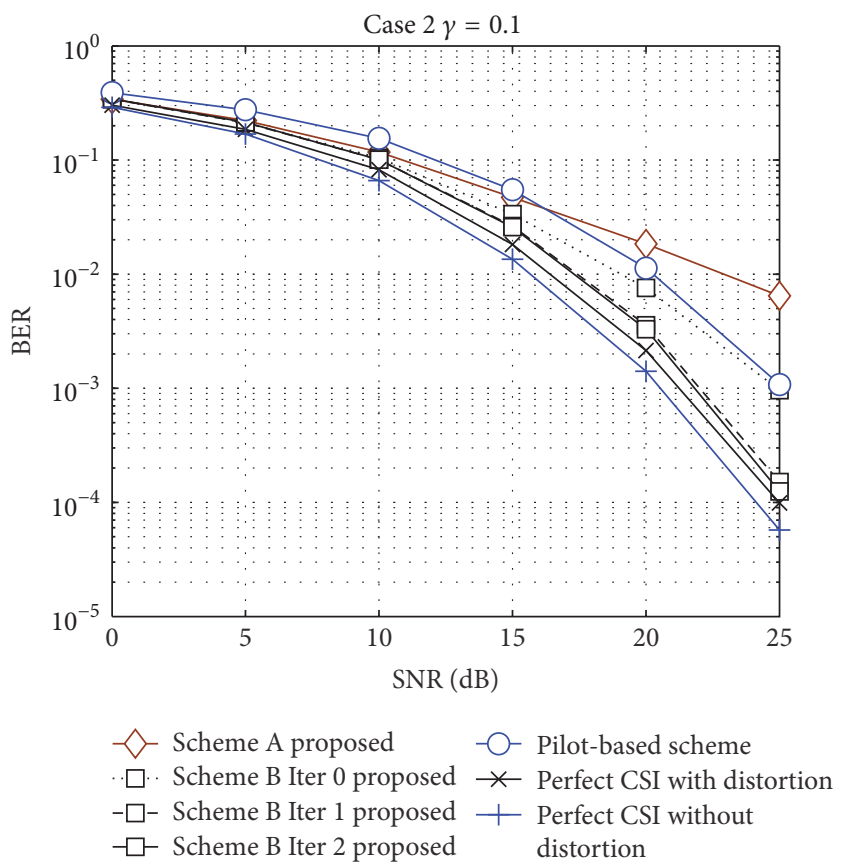

Figure 9: BER performance with different schemes for Case 2.

block $\left(N_{b}\right)$ increasing, the BER performance in the proposed scheme get a certain improvement. It is because that the distortion on every symbol is reduced with $N_{b}$ increasing, when the fixed distortion is scattered throughout the data block. As a result, when we employ the system parameter of larger frames, the proposed scheme is more competitive.

\section{Conclusions}

In the AF-based diamond relay network, a novel relayassisted training strategy is proposed to acquire the individual 
CSI. In our strategy, each relay node superimposes its own special training sequence over the amplified received data, which can be used to acquire the second-hop CSI. To solve the interference problems of unknown cooperative data, we discarded some cooperative data at each relay to accommodate the relay-pilot tones. Meanwhile, we derive a couple of relay training with autocorrelation and cross-correlation properties to decouple the combined relay training sequences. The simulations show that the channel estimation performance of the proposed scheme is superior to that of the pilotbased scheme described in [17]. Moreover, the detecting performance improvement via the iteration reconstruction is distinct.

\section{Conflicts of Interest}

The authors declare that they have no conflicts of interest.

\section{Acknowledgments}

This work was supported by the National Natural Science Foundation of China (Grant no. 61302099) and China Postdoctoral Science Foundation (Grant no. 2015T81107).

\section{References}

[1] W. Zhuang and M. Ismail, "Cooperation in wireless communication networks," IEEE Wireless Communications Magazine, vol. 19, no. 2, pp. 10-20, 2012.

[2] G. J. Foschini and M. J. Gans, "On limits of wireless communications in a fading environment when using multiple antennas," Wireless Personal Communications, vol. 6, no. 3, pp. 311-335, 1998.

[3] J. N. Laneman, D. N. Tse, and G. W. Wornell, "Cooperative diversity in wireless networks: efficient protocols and outage behavior," IEEE Transactions on Information Theory, vol. 50, no. 12, pp. 3062-3080, 2004.

[4] Q. Wang, P. Fan, M. R. McKay, and K. Ben Letaief, "On the position selection of relays in diamond relay networks," IEEE Transactions on Communications, vol. 59, no. 9, pp. 2515-2527, 2011.

[5] C. Huang and S. Cui, "On the alternative relaying gaussian diamond channel with conferencing links," IEEE Transactions on Wireless Communications, vol. 12, no. 2, pp. 758-768, 2013.

[6] A. Reznik, S. R. Kulkarni, and S. Verdú, "Degraded Gaussian multirelay channel: capacity and optimal power allocation," IEEE Transactions on Information Theory, vol. 50, no. 12, pp. 3037-3046, 2004.

[7] S. Zhang, F. Gao, C. Pei, and X. He, "Segment training based individual channel estimation in one-way relay network with power allocation," IEEE Transactions on Wireless Communications, vol. 12, no. 3, pp. 1300-1309, 2013.

[8] G. Yuan, M. Peng, and W. Wang, "Opportunistic user cooperative relaying in TDMA-based wireless networks," Wireless Communications and Mobile Computing, vol. 10, no. 7, pp. 972985, 2010.

[9] A. Bletsas, H. Shin, and M. Z. Win, "Cooperative communications with outage-optimal opportunistic relaying," IEEE Transactions on Wireless Communications, vol. 6, no. 9, pp. 3450-3460, 2007.
[10] Y. Jing and H. Jafarkhani, "Single and multiple relay selection schemes and their achievable diversity orders," IEEE Transactions on Wireless Communications, vol. 8, no. 3, pp. 1414-1423, 2009.

[11] M. Elfituri, A. Ghrayeb, and W. Hamouda, "Antenna/relay selection for coded cooperative networks with AF relaying," IEEE Transactions on Communications, vol. 57, no. 9, pp. 25802584, 2009.

[12] M. Lei, M. Zhao, J. Zhong, and Z. Zhang, "Channel estimation based on cyclic orthogonal training sequence for relayassisted cooperative communication system," in Proceedings of the International Conference on Computer, Information and Telecommunication Systems (CITS '12), Amman, Jordan, May 2012.

[13] J. Ma, P. Orlik, J. Zhang, and G. Y. Li, "Pilot matrix design for estimating cascaded channels in two-hop MIMO amplifyand-forward relay systems," IEEE Transactions on Wireless Communications, vol. 10, no. 6, pp. 1956-1965, 2011.

[14] O. Munoz-Medina, J. Vidal, and A. Agustin, "Linear transceiver design in nonregenerative relays with channel state information," IEEE Transactions on Signal Processing, vol. 55, no. 6, part 1, pp. 2593-2604, 2007.

[15] D. Qin, Z. Ding, and S. Dasgupta, "On forward channel estimation for MIMO precoding in cooperative relay wireless transmission systems," IEEE Transactions on Signal Processing, vol. 62, no. 5, pp. 1265-1278, 2014.

[16] H. Xi and A. L. F. de Almeida, "Multiuser receiver for joint symbol/channel estimation in dual-hop relaying systems," Wireless Personal Communications, vol. 83, no. 1, pp. 17-33, 2015.

[17] B. Zahedi, M. Ahmadian, K. Mohamed-Pour, M. Peyghami, M. Norouzi, and S. Salari, "Pilot-based individual forward and backward channel estimation in amplify-and-forward OFDM relay networks," in Proceedings of the IFIP Wireless Days (WD '11), Niagara Falls, Canada, October 2011.

[18] F. Gao, B. Jiang, X. Gao, and X. Zhang, "Superimposed training based channel estimation for OFDM modulated amplify-andforward relay networks," IEEE Transactions on Communications, vol. 59, no. 7, pp. 2029-2039, 2011.

[19] R. Simoni, V. Jamali, N. Zlatanov, R. Schober, L. Pierucci, and R. Fantacci, "Buffer-Aided Diamond Relay Network With Block Fading and Inter-Relay Interference," IEEE Transactions on Wireless Communications, vol. 15, no. 11, pp. 7357-7372, 2016.

[20] W. N. Yuan, P. Wang, and Z. P. Fan, "Enhanced performance for MIMO channel estimation based on implicit ZCZ training sequences," ACTA Electronica Sinica, vol. 38, no. 1, pp. 74-78, 2010.

[21] P. Z. Fan, N. Suehiro, N. Kuroyanagi, and X. M. Deng, "A class of binary sequences with zero correlation zone," IEEE Electronics Letters, vol. 35, no. 10, pp. 777-779, 1999. 


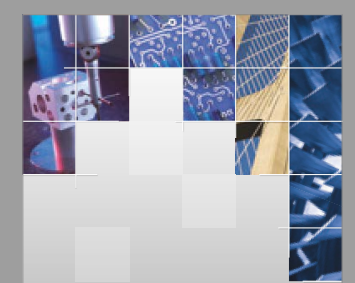

\section{Enfincering}
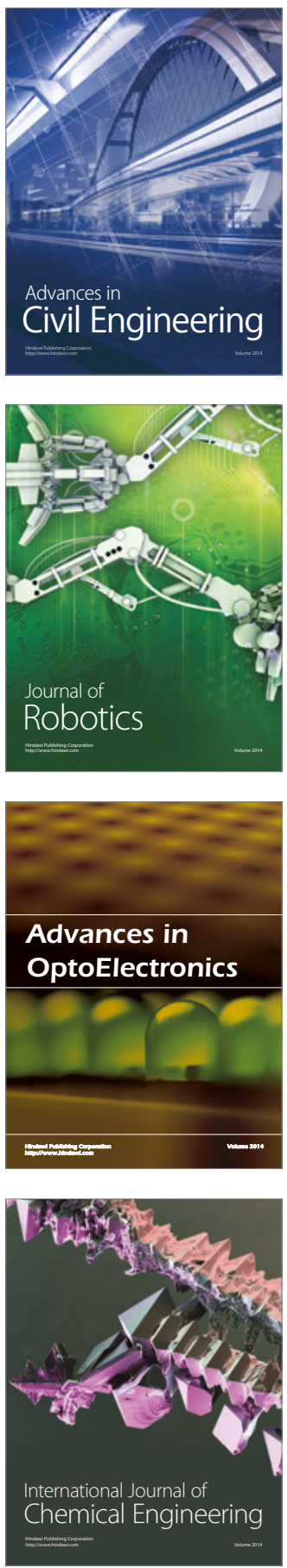

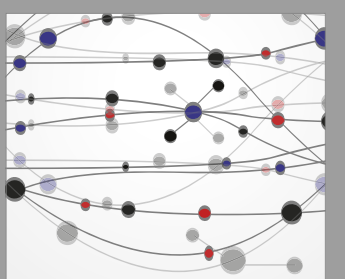

The Scientific World Journal

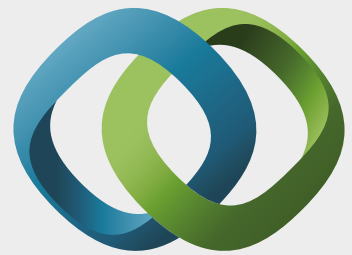

\section{Hindawi}

Submit your manuscripts at

https://www.hindawi.com
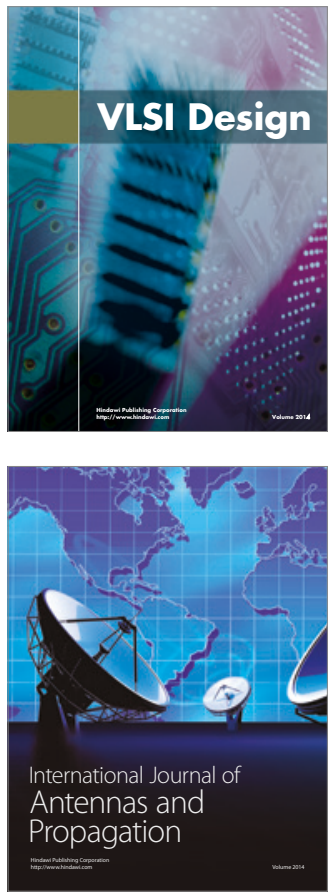

\section{Rotating}

Machinery
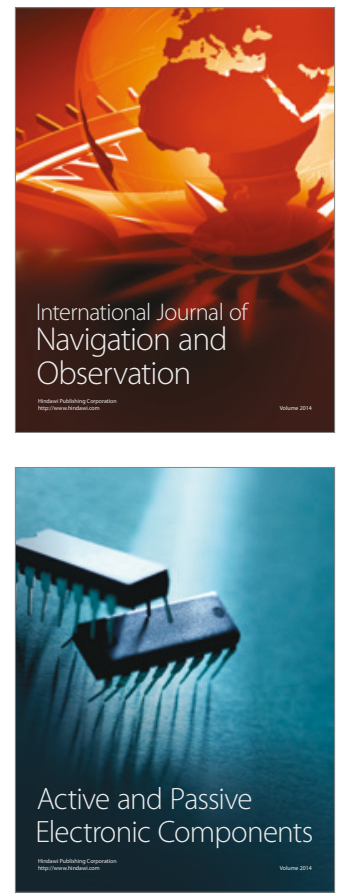
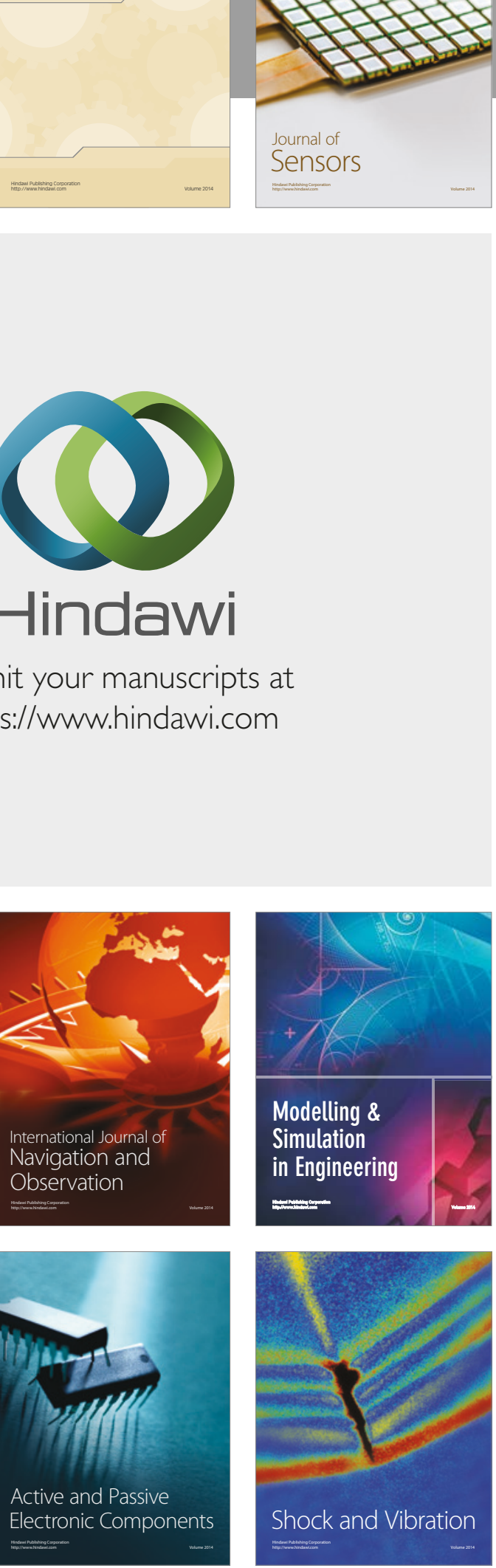
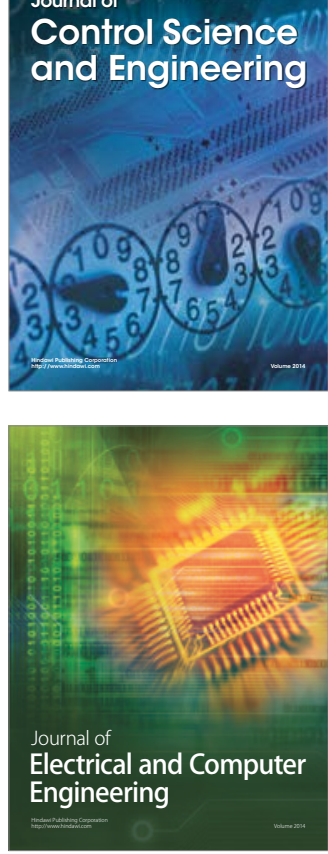

Distributed

Journal of

Control Science

and Engineering
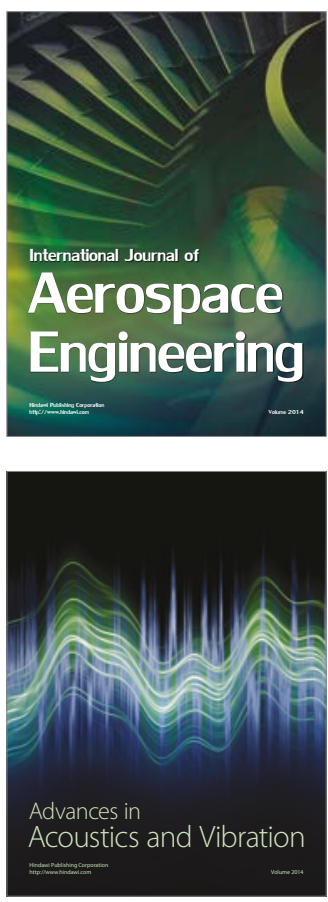

Sensor Networks 